\title{
Lattice Boltzmann model with generalized wall boundary conditions for arbitrary catalytic reactivity
}

\author{
Meysam Khatoonabadi $\odot,{ }^{*}$ Nikolaos I. Prasianakis $\odot,{ }^{\dagger}$ and John Mantzaras $\oplus^{\ddagger}$ \\ Paul Scherrer Institute, Laboratory for Scientific Computing and Modeling, and Waste Management Laboratory, \\ Nuclear Energy and Safety Division, CH-5232 Villigen PSI, Switzerland
}

(Received 22 December 2020; accepted 11 May 2021; published 3 June 2021)

\begin{abstract}
A lattice Boltzmann model for multispecies flows with catalytic reactions is developed, which is valid from very low to very high surface Damköhler numbers $\left(\mathrm{Da}_{\mathrm{s}}\right)$. The previously proposed model for catalytic reactions [S. Arcidiacono, J. Mantzaras, and I. V. Karlin, Phys. Rev. E 78, 046711 (2008)], which is applicable for lowto-moderate $\mathrm{Da}_{\mathrm{s}}$ and encompasses part of the mixed kinetics and transport-controlled regime, is revisited and extended for the simulation of arbitrary kinetics-to-transport rate ratios, including strongly transport-controlled conditions $\left(\mathrm{Da}_{\mathrm{s}} \rightarrow \infty\right)$. The catalytic boundary condition is modified by bringing nonlocal information on the wall reactive nodes, allowing accurate evaluation of chemical rates even when the concentration of the deficient reactant at the wall becomes vanishingly small. The developed model is validated against a finite volume NavierStokes CFD (Computational Fluid Dynamics) solver for the total oxidation of methane in an isothermal channelflow configuration. CFD simulations and lattice Boltzmann simulations with the old and new catalytic reaction models are compared against each other. The new model demonstrates a second order accuracy in space and time and provides accurate results at very high $\mathrm{Da}_{\mathrm{s}}\left(\sim 10^{9}\right)$ where the old model fails. Moreover, to achieve the same accuracy at moderate-to-high $\mathrm{Da}_{\mathrm{s}}$ of $O(1)$, the new model requires $\sim 2^{d} \times N$ coarser grid than the original model, where $d$ is the spatial dimension and $N$ the number of species.
\end{abstract}

DOI: 10.1103/PhysRevE.103.063303

\section{INTRODUCTION}

Chemical reactions and transport phenomena have wide applications in natural processes and industrial systems. These phenomena are important in corrosion, environmental contaminants, petroleum reservoirs, biology, as well as power generating devices such as combustion engines and fuel cells. Chemical reactions can be categorized into two major groups, namely, volumetric gas-phase (homogeneous) and surface catalytic (heterogeneous) reactions. Chemistry is typically coupled with diffusion and convection, rendering the underlying processes more complex. The presence of multiple chemical reactions, which occur in typical combustion applications at disparate temporal and spatial scales and high temperatures, renders fundamental experimental kinetic studies very challenging. For this reason, numerical methods have advanced in recent years that are able to predict realistic reacting systems under different operating conditions.

The lattice Boltzmann method (LBM) has been widely utilized for the simulation of numerous engineering flow problems [1,2]. Due to its mesoscopic nature, it is attractive for investigating multiphase and multicomponent (i.e., multispecies) flows. In particular, multicomponent flows have attracted increased attention in recent LBM modeling [3-7].

\footnotetext{
*seyyed-meysam.khatoonabadi@psi.ch

${ }^{\dagger}$ nikolaos.prasianakis@psi.ch

${ }^{\ddagger}$ Corresponding author: ioannis.mantzaras@psi.ch
}

One of the first attempts to simulate reactive flows using LBM was by Succi et al. [8]. They proposed an approach for simulating gas-phase chemical reactions, where the developed model was applicable for non-premixed combustion with very fast chemistry. Later on, Gabrielli et al. [9] employed LBM to study the effect of geometrical microirregularities on the catalytic reaction efficiency. They considered a simple obstacle with reactive surface in a channel and investigated the effects of geometry, chemistry, diffusion, and hydrodynamics. They included several species which were treated, similar to [1], as passive scalars. To study dissolution and precipitation, Kang et al. [2] proposed a new lattice Boltzmann (LB) model by considering appropriate Peklet and Damköhler numbers as the controlling nondimensional parameters. However, the simulation results were limited to geochemistry applications where solute concentration is low, such that a passive scalar approach was adopted for the chemical species.

He et al. [10] formulated the distribution function in order to incorporate a reaction-diffusion boundary condition. For this purpose, they considered that the soluble reactant is always in saturation state. Kang et al. [11] improved an earlier pore-scale model for the simulation of heterogeneous reactions. They utilized a passive scalar approach for convection-diffusion-reaction problems and compared two lattice structures for solute transport, the D2Q4 and the D2Q9. The root-mean-square deviation of the D2Q4 results was slightly higher than that of the D2Q9; however, the former was computationally more efficient. Furthermore, the D2Q9+D2Q5 passive scalar is an established and accurate model. However, for high Peclet number and flow velocities, 
the D2Q5 requires correction terms to recover accurately the advection diffusion equation. Li et al. [12] conducted a comparative study of D2Q5 and D2Q9 and showed that D2Q9 exhibits better accuracy, especially at high Peclet numbers.

Another class of multicomponent models, distinct from the passive scalar approach, is the kinetic theory based models, whereby each component has its own population with a corresponding concentration, velocity, and temperature. Such are the models by Arcidiacono et al. [13] and Kang et al. [14], where also a new catalytic boundary condition has been introduced. For consistency, these models need to recover as many higher order moments as possible and are therefore preferably constructed on the D2Q9 lattice. Simulation results of a two-dimensional isothermal channel flow with catalytic reactions, yielded very good agreement with results obtained from a standard reactive CFD code [13]. Moreover, the LB model was shown to be mass conserving.

Kang et al. [15] extended their previous passive scalar model [11] for multicomponent flows with heterogeneous and homogeneous reactions. They used a single relaxation time and the normal lattice Boltzmann equation. Nonetheless, some discrepancies were reported between their results and continuum-scale code results [15].

Most of the past studies focused on gas-phase reactions and flames. For example, Chiavazzo et al. [16] proposed a model for gas-phase reactive flows where a detailed chemical reaction mechanism and the lattice Boltzmann representation of the flow were coupled. Di Rienzo et al. [17] introduced a gas-phase reaction model among multiple species with high density and temperature variations. The extended model was capable of simulating low Mach number reactive flows.

In other reactive flows studies, Kang and Lichtner [18] extended their earlier model [15] to include homogeneous and heterogeneous reactions for a two-dimensional (2D) channel with reactive walls. The developed model showed accurate results; however, it was reported that the error was large at the channel inlet where the reaction rate was highest. Kang et al. [19] advanced the nonthermal multicomponent model by Arcidiacono et al. [13] to multicomponent thermal flows, such that each gaseous species had tunable Prandtl and Schmidt numbers. Although this model [19] was nonreactive, its thermal capacity paved the way for the subsequent development of realistic thermal and chemically reacting LB models with large temperature gradients (typically induced by the reaction exothermicity). They simulated [19] an opposed jet flow with high temperature gradients. Owing to theoretical considerations and the introduction of appropriate correction terms, the aforementioned model yielded very accurate results. Lin and Luo [20] introduced a model with three terms to account for chemical reaction, external force, and molecular collision. They investigated numerically premixed and non-premixed homogeneous combustion.

There are considerably fewer literature investigations on heterogeneous reactions by LBM, even though this topic is of main interest in many practical systems such as automotive exhaust gas treatment, fuel processing, synthesis of chemicals, power generation, geochemical applications [21,22], hydrogen recombiners in nuclear power plants [23], etc. Kang et al. [14] advanced the nonthermal multicomponent model with the catalytic reactive boundary condition from Arcidiacono et al. [13] to account for large temperature differences. The simulations [14] compared favorably to conventional CFD results, when considering a catalytic reaction with moderate reactivity. However, the performance and accuracy of this model [14] for high catalytic reactivity cases was not demonstrated. As will be elaborated in the next section, the original model [14] lacked a formal theoretical treatment for diffusionreaction systems in which diffusion played a significant role and could (in the case of transport-limited catalytic conversion) control the reactant consumption. Jv et al. [24] suggested a 2D boundary condition for the simulation of heterogeneous reactions, which displayed a second order accuracy for flat boundaries; however, for an inclined channel the accuracy was reduced. Recently, Kulyk et al. [25] utilized the catalytic reactive boundary condition from $[13,14]$ to study complex geometries with the addition of conjugate heat transfer in the solid. They simulated arrays of 2D squares, placed inside a channel, having catalytically reacting surfaces. It was reported that while the simulation of convex corners was straightforward, concave corners were challenging for this boundary condition.

In the present work, the previous reactive boundary condition by Arcidiacono et al. [13] is extended to cover a wide range of reaction-to-diffusion rates, spanning from kinetically controlled to diffusion-controlled (transport-limited) reactant conversion. In Sec. II, we introduce the LB model for a multicomponent gas mixture. Subsequently, the catalytic reactive boundary condition and its inherent limitations are presented in Sec. III. The herein developed reactive boundary condition is discussed in detail in Sec. IV, where a macroscopic nonlocal expression is incorporated into the catalytic reactive model. Afterwards, the original and the newly developed forms of the reactive boundary conditions are compared for cases with very low to very high catalytic reactivity (very low to very high Damköhler numbers). In Sec. V, the results of the new model are validated against reactive CFD code simulations and also compared to the original model. It is shown that the new model is second order accurate. Moreover, it is much more computationally efficient since there is no need to consider small lattice spacing and time step for large surface Damköhler numbers.

\section{MULTICOMPONENT LATTICE BOLTZMANN MODEL}

The two-dimensional multicomponent lattice Boltzmann model in [7] is adapted for three-dimensional (3D) configurations. The kinetic equation for each species is as follows:

$$
\begin{aligned}
\partial_{t} f_{j i}+c_{j i \alpha} \partial_{\alpha} f_{j i} & =-\frac{1}{\tau_{j 1}}\left(f_{j i}-f_{j i}^{*}\right)-\frac{1}{\tau_{j 2}}\left(f_{j i}^{*}-f_{j i}^{\mathrm{eq}}\right)+\psi_{j i}, \\
j & =1,2, \ldots, N, \quad i=0,1, \ldots, 26 .
\end{aligned}
$$

where $j$ is the index of species, with $N$ the total number of species, and $i$ is the discrete velocity indicator. Since a 3D lattice structure with 27 discrete velocities (D3Q27) is considered here, $i$ varies from 0 to 26 and $\alpha$ is 3 (corresponding to $x, y$, and $z$ directions). $\tau_{j 1}$ and $\tau_{j 2}$ are relaxation times linked to diffusion and viscosity of each component in the mixture. There are two equilibrium functions in Eq. (1): the quasiequilibrium $\left(f_{j i}^{*}\right)$ and equilibrium $\left(f_{j i}^{\mathrm{eq}}\right)$ which will be 
defined later. $\psi_{j i}$ is added in the right-hand side to satisfy momentum conservation up to second order and will be elaborated in the next section. Each species has its own discrete velocity $\left(c_{j i \alpha}\right)$, which is a function of its molar mass, and is written as ([26])

$$
\begin{aligned}
c_{j i x}= & c_{j}(0,1,0,-1,0,1,-1,-1,1,0,1,0,-1,0,1, \\
& -1,-1,1,0,1,0,-1,0,1,-1,-1,1), \\
c_{j i y}= & c_{j}(0,0,1,0,-1,1,1,-1,-1,0,0,1,0,-1,1,1, \\
& -1,-1,0,0,1,0,-1,1,1,-1,-1), \\
c_{j i z}= & c_{j}(0,0,0,0,0,0,0,0,0,1,1,1,1,1,1,1,1,1, \\
& -1,-1,-1,-1,-1,-1,-1,-1,-1),
\end{aligned}
$$

where $c_{j}$ indicates lattice velocities for every species with respect to the lightest species' molecular weight. If $M_{1}$ is the lightest species' molecular weight, $c_{j}$ is scaled as $\sqrt{M_{1} / M_{j}}$. The computational grid is based on the lightest species; therefore, it ensures that all species' populations stream by less than one lattice spacing at every time step.

The equilibrium distribution function $f_{j i}^{\mathrm{eq}}$ is obtained by minimizing the entropy function with two constraints, conservation of density and momentum [26,27]:

$$
\begin{aligned}
f_{j i}^{\mathrm{eq}}\left(\rho_{j}, u\right)= & \rho_{j} \prod_{\alpha=x, y, z} \frac{2 c_{0 i \alpha}^{2}-1}{2^{c_{0 i \alpha}^{2}}}\left[\left(c_{0 i \alpha}^{2}-1\right)\right. \\
& \left.+\sqrt{M_{j}} c_{0 i \alpha}^{2} u_{\alpha}+M_{j} u_{\alpha}^{2}+T\right],
\end{aligned}
$$

where $T$ is temperature and $c_{0 i}$ is calculated from Eq. (2) with $c_{0}=1$. The individual species densities $\rho_{j}=\sum_{i=0}^{i=26} f_{j i}$ and species momenta $J_{j \alpha}=\sum_{i=0}^{i=26} f_{j i} c_{j i \alpha}$ are first computed. The mixture density and momenta are $\rho=\sum_{j=0}^{j=N} \rho_{j}$ and $J_{\alpha}=$ $\sum_{j=0}^{j=N} J_{j \alpha}$, respectively. Therefore, the mixture velocity $U_{\alpha}$ is equal to $J_{\alpha} / \rho$. Another important parameter is the species concentration, defined as $C_{j}=\rho_{j} / M_{j}$, and the total concentration $C=\sum_{j=0}^{j=N} C_{j}$.

Similarly, the quasiequilibrium distribution function, constructed from the species velocities instead of the mixture velocity, is [26,27]

$$
\begin{array}{r}
f_{j i}^{*}\left(\rho_{j}, u_{j}\right)=\rho_{j} \prod_{\alpha=x, y, z} \frac{2 c_{0 i \alpha}^{2}-1}{2^{c_{0 i \alpha}^{2}}}\left[\left(c_{0 i \alpha}^{2}-1\right)\right. \\
\left.+\sqrt{M_{j}} c_{0 i \alpha}^{2} u_{j \alpha}+M_{j} u_{j \alpha}^{2}+T\right],
\end{array}
$$

where $U_{j \alpha}=J_{j \alpha} / \rho$.

The relaxation times relate to the binary diffusion and dynamic viscosity in Eq. (1). The mathematical derivations (using Chapman-Enskog expansions) have been given in previous works $[13,14]$. The relation between diffusion and relaxation times $\tau_{j 2}$ is obtained as [7]

$$
\tau_{j 2}=\frac{\rho_{j}}{P_{j}} D_{j m},
$$

where $P_{j}$ is the partial pressure of species $j$ and $D_{j m}$ is the mixture-averaged diffusion of each species which is calculated as ([28])

$$
D_{j m}=\frac{1-Y_{j}}{\sum_{k \neq j}^{N} X_{k} / D_{j k}},
$$

where $Y_{j}$ and $X_{j}$ are mass and mole fractions of species $j$, respectively, and $D_{j k}$ the binary diffusion coefficient of species $j$ and $k$. The mixture-averaged diffusion approximation does not lead to momentum conservation. In order to conserve momentum $J_{j \alpha}$, besides the calculated momentum of species $\tilde{J}_{j \alpha}$, a diffusion velocity correction $U_{\alpha}^{\mathrm{c}}$ is required such that $J_{j \alpha}=\tilde{J}_{j \alpha}+\rho_{j} U_{\alpha}^{\mathrm{c}}[7]$, leading to

$$
U_{\alpha}^{\mathrm{c}}=\frac{\sum_{j} \frac{1}{\tau_{j 2}}\left(\tilde{J}_{j \alpha}+\frac{\rho_{j}}{{ }_{i} \rho} J_{\alpha}\right)}{\sum_{j} \frac{\rho_{j}}{\tau_{j 2}}} .
$$

To add this velocity correction into the kinetic equation, we introduce the forcing term $\psi_{j i}$. The first part of this term relating to mixture-averaged diffusion is written as

$$
\Psi_{j i}^{\overline{1}}=\psi_{j i \alpha} \frac{\rho_{j} U_{\alpha}^{\mathrm{c}}}{\tau_{j 2}},
$$

where $\Psi_{j i \alpha}$ are the following coefficients [26]:

$$
\begin{aligned}
\psi_{j i x}= & \frac{1}{c_{j}}\left(0, \frac{3}{2}, 0,-\frac{3}{2}, 0,-\frac{3}{8}, \frac{3}{8}, \frac{3}{8},-\frac{3}{8}, 0,-\frac{3}{8}, 0, \frac{3}{8}, 0,\right. \\
& \left.\frac{1}{8},-\frac{1}{8},-\frac{1}{8}, \frac{1}{8}, 0,-\frac{3}{8}, 0, \frac{3}{8}, 0, \frac{1}{8},-\frac{1}{8},-\frac{1}{8}, \frac{1}{8}\right), \\
\psi_{j i y}= & \frac{1}{c_{j}}\left(0,0, \frac{3}{2}, 0,-\frac{3}{2},-\frac{3}{8},-\frac{3}{8}, \frac{3}{8}, \frac{3}{8}, 0,0,-\frac{3}{8}, 0, \frac{3}{8},\right. \\
& \left.\frac{1}{8}, \frac{1}{8},-\frac{1}{8},-\frac{1}{8}, 0,0,-\frac{3}{8}, 0, \frac{3}{8}, \frac{1}{8}, \frac{1}{8},-\frac{1}{8},-\frac{1}{8}\right), \\
\psi_{j i z}= & \frac{1}{c_{j}}\left(0,0,0,0,0,0,0,0,0, \frac{3}{2},-\frac{3}{8},-\frac{3}{8},-\frac{3}{8},-\frac{3}{8},\right. \\
& \left.\frac{1}{8}, \frac{1}{8}, \frac{1}{8}, \frac{1}{8},-\frac{3}{2}, \frac{3}{8}, \frac{3}{8}, \frac{3}{8}, \frac{3}{8},-\frac{1}{8},-\frac{1}{8},-\frac{1}{8},-\frac{1}{8}\right),
\end{aligned}
$$

The second part of the correction term $\left(\Psi_{j i}^{\overline{2}}\right)$ defines the deviation term in the pressure tensor $\left(\partial_{\beta} P_{\alpha \beta}^{\prime \prime}\right)$ from the macroscopic momentum equation (see [14]) and is

$$
\Psi_{j i}^{\overline{2}}=\psi_{j i \alpha} \partial_{\beta} P_{\alpha \beta}^{\prime \prime} .
$$

Finally, the total correction term $\Psi_{j i}$ is the summation of the two aforementioned terms [14]:

$$
\Psi_{j i}=\Psi_{j i}^{\overline{1}}+\Psi_{j i}^{\overline{2}} \text {. }
$$

The other relaxation times $\tau_{j 1}$ are linked to the species' viscosity. The individual species dynamic viscosities are $\mu_{j}$ and the dynamic viscosity of the mixture $\mu$ is defined as [14]

$$
\mu=\sum_{j}^{N}\left(\tau_{j 1} C_{j} T\right) .
$$

On the other hand, the empirical Wilke formula [29] for the dynamic viscosity of a mixture as a function of concentration, viscosity, and molecular weight is

$$
\mu=\sum_{j}^{N} \frac{X_{j} \mu_{j}}{\sum_{k}^{N} X_{k} \varphi_{j k}},
$$


in which $\varphi_{j k}$ is a function of the molecular masses and species' dynamic viscosities

$$
\varphi_{j k}=\frac{1}{\sqrt{8}}\left(1+\frac{M_{j}}{M_{k}}\right)^{-1 / 2}\left[1+\left(\frac{\mu_{j}}{\mu_{k}}\right)^{1 / 2}\left(\frac{M_{k}}{M_{j}}\right)^{1 / 4}\right]^{2} .
$$

From Eqs. (13) and (14), the relaxation times $\tau_{j 1}$ are obtained as

$$
\tau_{j 1}=\frac{\mu_{j}}{p \sum_{k}^{N} X_{k} \varphi_{j k}} .
$$

Since the grid size is based on the lightest species, all other species propagate relative to the chosen grid size in the streaming step. However, an interpolation scheme is required to calculate the value on the nearest node. To keep the second order accuracy of the model, we used the same interpolation scheme as in [13].

\section{CATALYTIC REACTIVE BOUNDARY CONDITION}

The diffusive boundary condition [30] according to which the incoming populations into the domain are redistributed to produce the outgoing populations was modified by Arcidiacono et al. [13] to incorporate a catalytic reaction at the boundary. Since the model should be totally (in terms of all species $j$ ) mass conserving, the incoming mass flux $\left(\phi_{j}^{\text {in }}\right)$ to the domain is equal to the outgoing mass flux $\left(\phi_{j}^{\text {out }}\right)$ plus the mass reaction rate

$$
M_{j} \dot{S_{j}}=\phi_{j}^{\text {in }}-\phi_{j}^{\text {out }} .
$$

We can rewrite Eq. (16) in terms of populations [13]

$$
\begin{aligned}
M_{j} \dot{S}_{j}= & \sum_{i=0, f_{j i c} c_{j i \alpha} n_{\alpha}>0}^{i=26}\left|f_{j i} c_{j i \alpha} n_{\alpha}\right| \\
& -\sum_{i=0, f_{j i} c_{j i \alpha} n_{\alpha}<0}^{i=26}\left|f_{j i} c_{j i \alpha} n_{\alpha}\right| .
\end{aligned}
$$

In Eq. (17), $n_{\alpha}$ is the local normal vector to the catalytic surface; positive and negative values of $f_{j i} c_{j i \alpha} n_{\alpha}$ indicate incoming and outgoing fluxes, respectively. In Eqs. (16) and (17), $S_{j}$ denotes the molar surface reaction rate. The schematic in Fig. 1 represents one LB time step including collision, streaming, and the current full-way reactive boundary condition for a D2Q9 model (the 3D extension is straightforward). All populations $f_{i}$ are categorized into three groups, i.e., incoming populations from the reactive surface to the fluid domain, remaining (rest) populations which are not altered by the catalytic reactive boundary condition, and outgoing populations leaving the domain due to reaction. It is noted that all populations parallel to the reactive surface $\left(f_{j i}^{\text {rest }}\right)$ remain unchanged during catalytic boundary condition [see Fig. 1(c)].

The total incoming flux $\phi_{j}^{\text {in }}$ is solved through Eq. (17). Then, we need to partition the total incoming flux to incoming populations in each $i$ direction. The population partitioning follows the diffusive boundary condition of Ansumali et al. [30]. Assuming Maxwellian velocity distribution, whereby fluid molecules have enough time to equilibrate with the

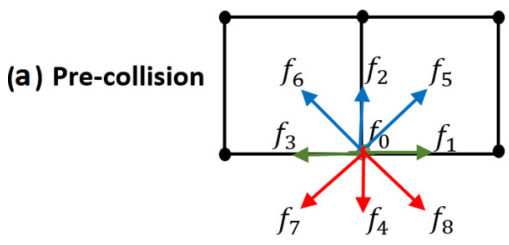

(b) Post-collision
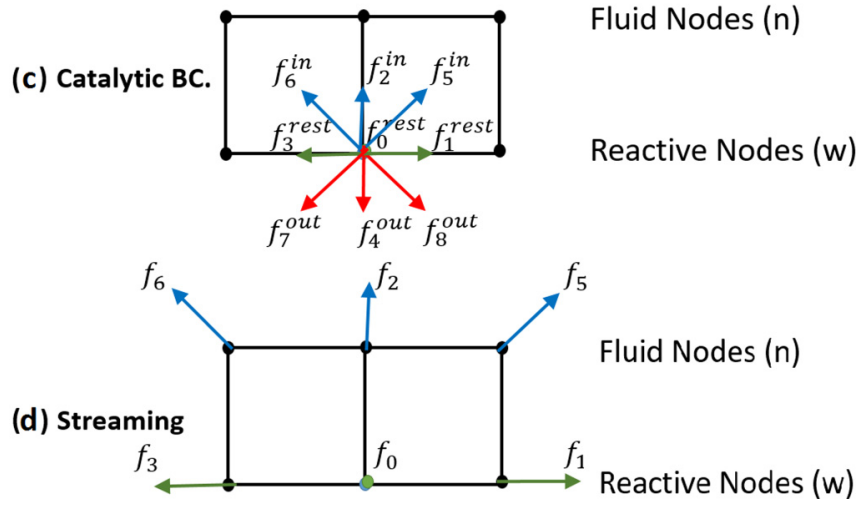

FIG. 1. The full-way reactive boundary condition steps.

wall temperature, the incoming populations can be calculated as in [13]:

$$
f_{j i}=f_{j i}^{\mathrm{eq}}\left(\rho=1, U_{w}\right) \frac{\sum_{i=0, f_{j i} c_{j i \alpha} n_{\alpha}<0}^{i=26}\left|f_{j i} c_{j i \alpha} n_{\alpha}\right|+M_{j} \dot{S}_{j}}{\sum_{i=0, f_{j i} c_{j i \alpha} n_{\alpha}>0}^{i=26}\left|f_{j i}^{\mathrm{eq}}\left(\rho=1, U_{w}\right) c_{j i \alpha} n_{\alpha}\right|},
$$

where the numerator is the total incoming flux obtained from Eq. (17) and $U_{w}$ is the velocity of the reactive wall. The catalytic reaction rate $\dot{S}_{j}$ is positive for products and negative for reactants. One common approach to calculate the catalytic reaction rate is an irreversible reaction of the type $b_{1} A+b_{2} B \rightarrow$ $b_{3} C+b_{4} D$, with $A$ and $B$ denoting the reactants and $C$ and $D$ the products. Without loss of generality, the reaction rate is considered first order with respect to the deficient reactant and zero order with respect to the other reactant. This is a realistic description for many practical reactions, such as the total oxidation of hydrocarbons or the hydrogen oxidation on noble metals, where the order $n$ is indeed unity with respect to the deficient fuel and zero with respect to the excess oxygen [31-33]. If the deficient reactant is species number 1 , then

$$
\dot{S}_{j}=b_{j} k C_{1 w}^{n},
$$

where $S_{j}$ is the absolute value of the molar surface reaction rate $\left(\mathrm{mol} / \mathrm{cm}^{2} \mathrm{~s}\right), b_{j}$ is the stoichiometric coefficient of species $j, k$ is the reaction rate coefficient, $C_{1 w}$ is the concentration $\left(\mathrm{mol} / \mathrm{cm}^{3}\right)$ of the first species at the gas-wall interface, and the reaction order is $n=1$. 
The reaction rate coefficient is a function of wall temperature $T_{w}$ and activation energy $E_{a}$ :

$$
k=A \exp \left(\frac{-E_{a}}{R T_{w}}\right)
$$

with $R$ the universal gas constant. $k$ or $A$ in Eqs. (19) and (20) have units of $\mathrm{cm} / \mathrm{s}$ for the adopted first order reaction.

All simulations are isothermal, with a constant wall temperature equal to the bulk gas temperature; such a condition is approached, for example, in automotive exhaust gas treatment catalysts. The reaction rate is related to the concentration of the first component at the gas-wall interface $C_{1 w}$, which is unknown at this stage. To solve this problem in the original catalytic reaction LB model $[13,14]$, the initial guess for $C_{1 w}$ at every time step is taken from the neighbor fluid node's concentration [see Fig. 1(a)]; an iterative procedure is then applied at each time step to correct the initial guess and reach a converged solution satisfying Eqs. (18) and (19). It is noted that the boundary condition in Eq. (17) is completely local, involving properties at the on-wall reactive node only.

This model has been validated for the total oxidation of methane [13], when the reaction rate is lower or comparable to the mass transport (diffusion) rate. It is worth mentioning that high or low reaction rates are generally determined by the dimensionless catalytic Damköhler number, defined as $\mathrm{Da}_{\mathrm{s}}=\frac{(h / 2) k}{D}$ where $h$ is the height of the catalytic channel and $D$ the diffusion coefficient of the deficient reactant [34]. For isothermal conditions with a given channel geometry and uniform inlet species concentrations, we can control the catalytic reactivity by changing the preexponential $A$ in the Arrhenius expression of Eq. (20).

The original model [13] was proposed as a half-way boundary condition, requiring two extrapolations in order to calculate the values on a reactive wall placed $\Delta x / 2$ from the last fluid node. However, we have adopted here a full-way treatment of the boundary condition (see Fig. 1) that is generally more convenient to implement [35].

To understand the potential limitations of the original model [13], we should bring out the underlying physics behind reaction and diffusion, and consider a wide range of reaction rates (from very low to very high), which can occur in practical systems. Figure 2 shows the transverse mass fraction profiles of the deficient reactant for different surface Damköhler numbers $\mathrm{Da}_{\mathrm{s}}$, at a given cross section of a $2 \mathrm{D}$ channel. The results are obtained using our finite difference CFD code [32] for the isothermal total oxidation of methane $\left(\mathrm{CH}_{4}+2 \mathrm{O}_{2} \rightarrow \mathrm{CO}_{2}+2 \mathrm{H}_{2} \mathrm{O}\right)$. Species transport properties were calculated from the Chemkin transport package [36]. The inlet reactant concentrations and the inlet velocity are the same in all simulations and only the reaction rate, which is first order with respect to the deficient methane reactant, is varied.

When the Damköhler number is small $\left[O\left(10^{-2}\right)\right]$, which signifies a slow catalytic reaction, the system is controlled by kinetics. That is, the species production and consumption is controlled by the chemical reaction rate. An increase in the catalytic reactivity over the range $0.01<\mathrm{Da}_{\mathrm{s}}<20$ leads to mixed reaction-diffusion controlled reactant conversion (see Fig. 2). For very high reactivity $\left(\mathrm{Da}_{\mathrm{s}} \geqslant 300\right.$ in Fig. 2$)$, the

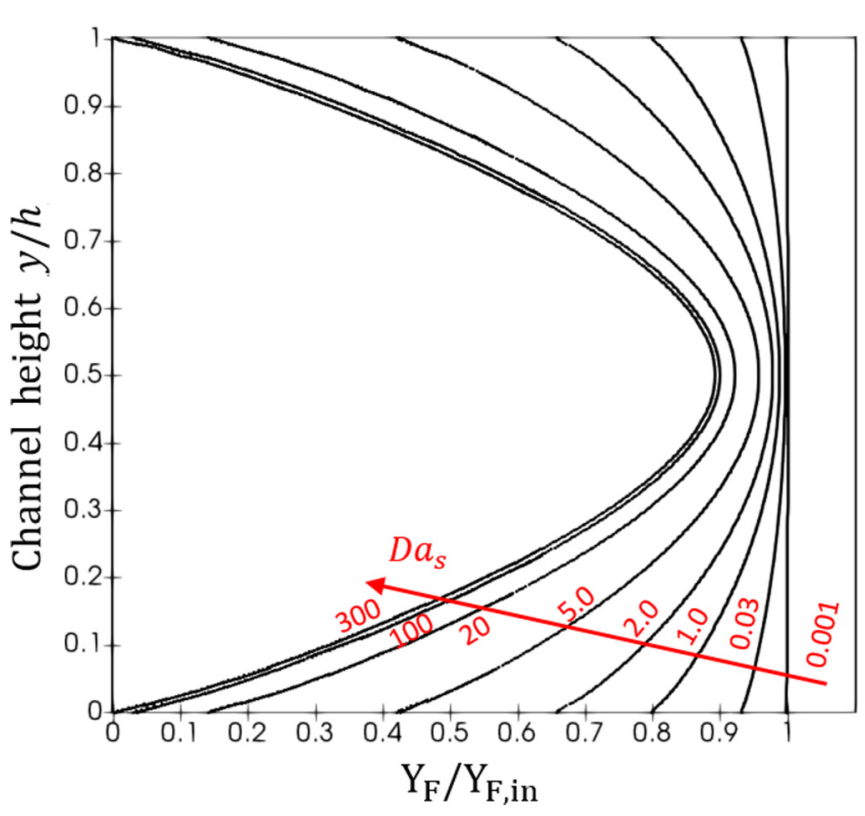

FIG. 2. The effect of Damköhler number on transverse mass fraction profiles at a given cross section $(x=0.2 \mathrm{~mm})$ of a $2 \mathrm{D}$ channel $(0.5 \mathrm{~mm} \times 5 \mathrm{~mm})$ obtained from CFD simulations: $U_{\mathrm{in}}=$ $7.2 \mathrm{~m} / \mathrm{s}, X_{\mathrm{in}, \mathrm{CH}_{4}}=0.1, X_{\mathrm{in}, \mathrm{O}_{2}}=0.9 . T_{\text {in }}=T_{\text {wall }}=\mathrm{const}=1200 \mathrm{~K}$, $P=1$ bar. $Y_{F}$ indicates the mass fraction of the deficient species (methane).

species chemical conversion is not controlled by kinetics but by diffusion (in the limit of infinite $\mathrm{Da}_{\mathrm{s}}$, the reactant consumption is termed transport limited). Note that as $\mathrm{Da}_{\mathrm{s}}$ increases above 300, the mass fraction of the deficient reactant at the wall drops sharply (approaching asymptotically to zero as $\mathrm{Da}_{\mathrm{s}} \rightarrow \infty$ ), such that the transverse species profiles in Fig. 2 remain practically unaltered. The strong dependence of the transverse species profiles on $\mathrm{Da}_{\mathrm{s}}$ in the mixed kinetics-diffusion controlled regime $0.01<\mathrm{Da}_{\mathrm{s}}<20$ has led to the advancement of laser-based techniques for measuring species concentration transverse profiles inside catalytic channels (e.g., 1D Raman spectroscopy) that have in turn allowed for direct assessment of the Damköhler number $\mathrm{Da}_{\mathrm{s}}$ and hence of the catalytic reactivity [37].

It has been further shown [34] that the profiles in Fig. 2 become universal when parametrized in terms of $\mathrm{Da}_{\mathrm{s}}$ and the inlet Reynolds number $\mathrm{Re}_{\mathrm{in}}$, when the axial and transverse independent variables are transformed to the inverse Graetz number $\zeta=x /\left[(h / 2) \operatorname{Re}_{\text {in }} \operatorname{Pr}\right]$ and $\eta=\frac{\int_{0}^{y} \rho d y}{(h / 2) \rho_{\text {in }} \sqrt{2 \operatorname{Pr} \zeta}}$, respectively, and when the mixture Prandtl number Pr and the deficient reactant's Schmidt number Sc are constant. Figure 3 provides universal plots for the wall mass fraction gradient and the wall mass fraction of the deficient reactant at the operating conditions of Fig. 2 (corresponding to a normalized axial distance $\zeta=0.12$ ). Such plots reveal that for $\mathrm{Da}_{\mathrm{s}}>300$ (shaded area in Fig. 3) the normalized wall mass fraction gradient changes by less than $0.3 \%$ but the normalized wall mass fraction changes by eight orders of magnitude, reaching values as low as $4.5 \times 10^{-10}$ for $\mathrm{Da}_{\mathrm{s}}=10^{10}$. The near constancy of the species gradient for $\mathrm{Da}_{\mathrm{s}}>300$ is equivalent to the near constancy of the reaction rate $S_{j}$, since the interfacial 


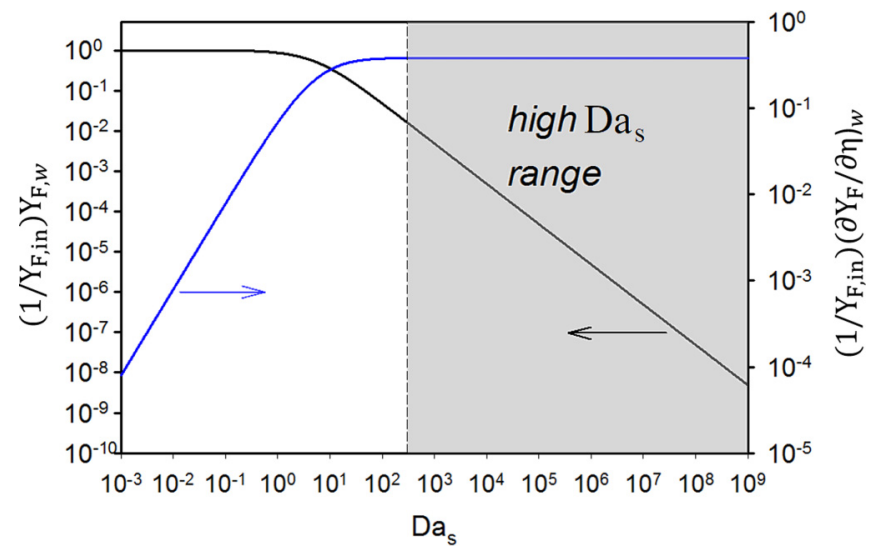

FIG. 3. Normalized deficient species wall gradient of mass fraction and wall mass fraction as a function of the catalytic Damköhler number $\mathrm{Da}_{\mathrm{s}}$ for the conditions of Fig. 2.

species boundary condition is

$$
D_{j m}\left(\frac{\partial C_{j}}{\partial y}\right)_{w}=S_{j}=b_{j} k C_{1 w}
$$

The implications are that when calculating the local reaction rate at very high $\mathrm{Da}_{\mathrm{s}}$ directly from Eq. (19), very high accuracy is required for the wall mass fraction (which is linked to the wall concentration as $\left.Y_{j, w}=C_{j, w} M_{j} / \rho_{w}\right)$ since we must satisfy $C_{j, w} \rightarrow 0$ as $k \rightarrow \infty$ in a way such that the product $k C_{j, w}$ remains constant and finite. In standard CFD codes, this difficulty is removed by simultaneously satisfying the species boundary conditions, Eq. (21), since the gradient $\left(\frac{\partial C_{j}}{\partial y}\right)_{w}$ brings nonlocal information from the near-wall node.

The model of Eq. (18), since it is based on local quantities at the reactive wall, suffers from the same issues of accuracy at very high reaction rates described before. This is manifested by a negative numerator in Eq. (18) when $\left|\sum_{i=0, f_{j i} c_{j i \alpha} n_{\alpha}<0}^{i=26}\right| f_{j i} c_{j i \alpha} n_{\alpha}||<\left|M_{j} \dot{S}_{j}\right|$, causing either divergence or incorrect mass production and consumption. To prevent any negative population, the grid size must be refined proportionally to the increased reaction rate $\left(\right.$ or $\left.\mathrm{Da}_{\mathrm{s}}\right)$. Furthermore, the reaction rate value is different from Eq. (19), especially at the initial time steps, as will be clarified in Sec. V. Accordingly, the calculation should be performed in a consistent way by invoking the proper physics of nonlocal contributions into Eq. (18) in order to have a general form of boundary condition applicable to all reaction conditions.

\section{DEVELOPMENT OF CATALYTIC BOUNDARY CONDITION}

As discussed in the foregoing section, although the original model shows accurate results for low and moderate catalytic
Damköhler numbers $\left(\mathrm{Da}_{\mathrm{s}}<1\right)$, it cannot provide good predictions at higher Damköhler numbers.

In order to calculate the exact concentration on the reactive wall based on the reaction rate coefficient and the diffusion coefficient, macroscopic equations are employed as well as mass conservation. The mass conservation is already employed in Eq. (18); however, mass transfer is not satisfied explicitly by the previous equations. For this purpose, we define another equation as

$$
\frac{D_{1 m}\left(C_{1 n}-C_{1 w}\right)}{\Delta y}=\dot{S}_{1},
$$

where $C_{1 w}$ and $C_{1 n}$ are the concentrations of the first species at the wall and the neighbor node, respectively (see Fig. 1), $y$ is the normal direction to the reactive surface, and $\Delta y$ is equal to unity in LB. Equation (22) is the discretization of Eq. (21). Consequently, the combination of Eqs. (19) and (22) ends up with

$$
\frac{D_{1 m}\left(C_{1 n}-C_{1 w}\right)}{\Delta y}=b_{1} k C_{1 w}^{n} .
$$

Equation (23) is an additional expression for the calculation of $C_{1 w}$. Equation (23) can be solved iteratively for $C_{1 w}$ when $n \neq 1$ since all other parameters are known. Hence, we do not need any guess for the concentration at the wall and the corresponding reaction rate in the LBM algorithm. It is more convenient to discuss the model when $n=1$, since as mentioned before this is a realistic assumption for many applications.

Considering $n=1$ and using Eq. (23), the following expression is obtained:

$$
\dot{S}_{1}=\frac{C_{1 n}}{\frac{\delta y}{D_{1 m}}+\frac{1}{b_{1} k}} .
$$

Equation (24) is the new proposed form of Eq. (19) in which diffusion mass transfer is explicitly utilized. For all other species, we have $\dot{S}_{j}=b_{j} k C_{1 w}$.

The advantage of Eq. (24) over Eq. (19) is that the reaction rate calculated by Eq. (24) cannot become unbounded since as $k \rightarrow \infty$ the second term in the denominator of Eq. (24) approaches zero while the first term remains constant and bounded. Therefore, it is also valid for transport-limited reactions $\left(\mathrm{Da}_{\mathrm{s}} \rightarrow \infty\right)$. We can show that the concentration of the first component (deficient reactant) decreases to zero when $\mathrm{Da}_{\mathrm{s}} \rightarrow \infty$. From Eq. (23), the first species concentration on the wall can be directly solved as

$$
C_{1 w}=\frac{C_{1 n}}{\frac{b_{1} \delta y k}{D_{1 m}}+1.0} .
$$

Therefore, irrespective of the concentration of the neighbor node $C_{1 n}, C_{1 w}$ approaches zero when $k \rightarrow \infty$.

To calculate the population at every given direction, Eq. (18) is used with the new form of reaction rate obtained in Eq. (24). Finally, the populations $f_{j i}$ are calculated as

$$
f_{j i}=f_{j i}^{\mathrm{eq}}\left(\rho=1, U_{w}\right) \frac{\sum_{i=0, f_{j i} c_{j i \alpha} n_{\alpha}<0}^{i=26}\left|f_{j i} c_{j i \alpha} n_{\alpha}\right|+\frac{b_{j} M_{j} C_{1 n}}{b_{1} \delta}+\frac{1}{D_{1 m}}+\frac{1}{k}}{\sum_{i=0, f_{j i} c_{j i \alpha} n_{\alpha}>0}^{i=26}\left|f_{j i}^{\mathrm{eq}}\left(\rho=1, U_{w}\right) c_{j i \alpha} n_{\alpha}\right|} .
$$


The new boundary condition in Eq. (26), which is constructed by including the one-sided finite difference approximation of the normal concentration into a fully kinetically consistent boundary condition for multicomponent reactive flows, is applicable for arbitrary $\mathrm{Da}_{\mathrm{s}}$ (from zero to practically infinity). Furthermore, being kinetically consistent, this boundary condition can handle arbitrary reactant concentrations in the bulk flow, including a consistent feedback and effect of the concentration gradients to the flow field. Equation (26) is written in a general form in terms of incoming and outgoing populations and can be directly applied on straight walls as well as on concave and convex corners, by replacing the $y$ coordinate with the local normal to the surface. The number of incoming and outgoing populations in concave and convex corners will of course be different from those of a straight wall. The derivation of such populations for curved boundaries is beyond the scope of this paper. It is further noted that straight channels are not a convenient idealization, but are used in both laboratory and industrial catalytic systems (see Refs. [23,32-34,37,38]).

There are two main differences between the new calculated reaction rate and the one used in Eq. (19). First, the effect of diffusion coefficient is explicitly included in the new expression. This brings the following advantages: Eq. (24) always ensures a positive numerator even if the reaction rate coefficient is several orders of magnitude larger than the diffusivity. In this case, increasing the reaction coefficient cannot lead to infinite consumption or production, since $1 / D_{1}$ is constant and nonzero. Therefore, transport-limited operation can be simulated through the new equation. Moreover, it satisfies the macroscopic diffusion-reaction equation [i.e., Eq. (23)]. Second, the reaction rate is a function of known concentrations, consequently, no initial guess and iterations are needed to calculate concentration at the wall. It is noted, however, that Eq. (23) for a nonunity reaction order $(n \neq 1)$ requires an iterative solution.

Although the bulk 3D LB gas flow model in the present work is isothermal, the developed catalytic reaction boundary condition in Eq. (26) is not limited to isothermal systems. This is similar to our previous works, where in Arcidiacono et al. [13] the old catalytic boundary condition was developed and applied to 2D isothermal gas flows while later in Kang et al. [14] the same catalytic boundary condition was applied to 2D thermal bulk gas flows. However, a 3D thermal multicomponent LB model would require additional correction terms in the LB model itself (and not in the catalytic boundary condition), but their derivation is beyond the scope of this paper.

\section{RESULTS AND DISCUSSION}

In this section, we present simulation results for a channel with catalytic boundary condition. The results are first validated against those of a CFD code and also compared to the original model at steady state. Then the preexponential $A$ of the reaction rate coefficient is altered such that the Damköhler number changes from very small (kinetically controlled conversion) to very large (transport-limited conversion). At the end, the mole fractions of species at a given channel cross section are compared for the two LB models for transient simulation.
The employed CFD tool is a steady Navier-Stokes reactive code, capable of handling catalytic (heterogeneous) and gaseous (homogeneous) chemical reactions, where the governing set of equations is discretized using a finite volume approach. A SIMPLER (Semi-Implicit Method for Pressure Linked Equations Revised) method is employed for the pressure-velocity field and solution is obtained iteratively using an ADI (Alternating-Direction Implicit) algorithm (details in [38]). An orthogonal staggered grid of $300 \times 60$ points (in $x$ and $y$, respectively, for the $5 \mathrm{~mm} \times 0.5 \mathrm{~mm}$ channel domain, with uniform $x$ spacing and finer $y$ spacing towards both walls), is sufficient to produce a grid-independent solution. The application of the catalytic boundary condition for the solution of the surface concentration of the deficient reactant is achieved with a modified Newton method using SURFACE CHEMKIN [39]. Local transport properties (species diffusivities and viscosities) are evaluated from the CHEMKIN transport package [36].

We utilize a 3D lattice Boltzmann multicomponent gas mixture parallel code by taking advantage of hybrid message passing interface (MPI) and general-purpose computing on graphics processing units (GPGPU). In contrast to the previous studies $[13,14]$ where the calculation of transport properties, i.e., viscosity and diffusion at every node in the domain was accomplished by coupling the code to the CHEMKIN package $[36,39]$, we now carry out these calculations directly on the GPU, and the LB code, by including appropriate routines. The main reason is that coupling a CUDA code with an external package running on CPUs slows down the algorithm appreciably.

In spite of the nonlocality of the proposed model, the numerical algorithm is not computationally more expensive since the other main steps including collision and streaming remain intact and only reactive nodes are affected. Moreover, in contrast to the original model, the proposed model needs no iteration to calculate the reactive node concentration, thus rendering it faster. Regarding the parallelization efficiency, while the scheme is nonlocal, it requires information only from the next neighboring nodes. For the specific GPU code used here, as well as for most of the parallel LB codes, the next neighbor populations belong to the Halo exchange zones and are readily available and exchanged at every time step anyway (Halo zones: overlapping population borderline communication zones between neighboring computational blocks, defined during the domain decomposition).

In order to have a meaningful comparison with previous studies, the total oxidation of methane is considered, as in $[13,14]$, whereby $\mathrm{CH}_{4}$ is the deficient reactant. The simulation setup for the channel is shown in Fig. 4. Initial mole fractions in the domain are the same as the inlet values. The inlet velocity is uniform, equal to $7.2 \mathrm{~m} / \mathrm{s}$. The physical channel length $(L)$ is $5 \mathrm{~mm}$ and the channel height $(h)$ is $0.5 \mathrm{~mm}$. The gas and wall temperatures are $1200 \mathrm{~K}$ and constant during simulation. The inlet boundary condition is set as Dirichlet, while for the outlet boundary condition, second order Neumann is employed.

Due to higher reaction rates in most simulations, we observed that a finer grid (compared to the previous studies $[13,14])$ is required. Therefore, in most simulations, the height and length of the channel are discretized into 400 and 4000 


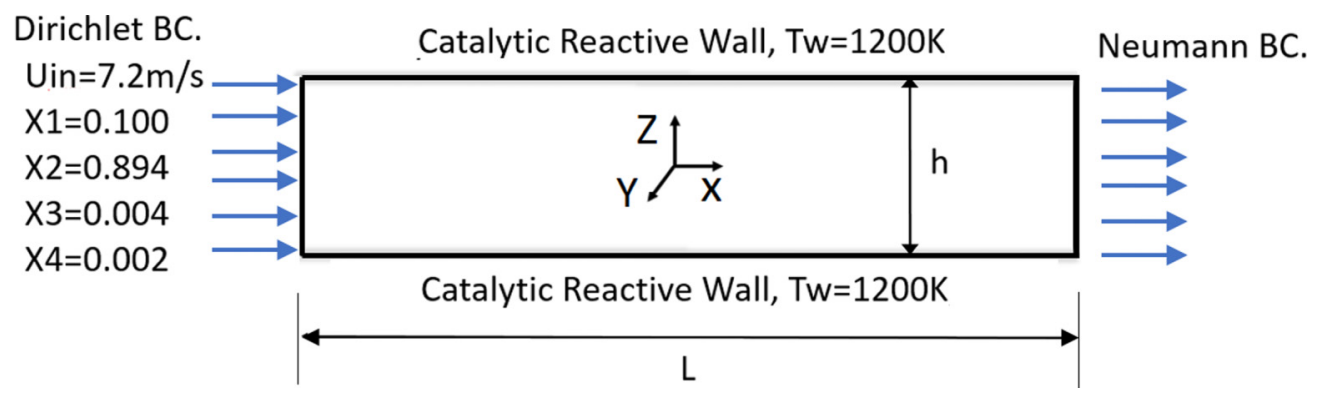

FIG. 4. Schematic of the channel geometry with reactive walls and boundary conditions.

grids, respectively. To reduce the three-dimensional domain into a two-dimensional one and compare the results with the results of the 2D CFD code, only five nodes are considered in the $y$ direction with periodic boundary conditions.

The reactive boundary condition proposed by Arcidiacono et al. [13] is a second order scheme. To assess the order of convergence of the present model, the simulation time step was reduced, while keeping other settings constant. We consider a lattice with an eightfold finer time step (and finer grid) as a reference simulation and calculate the error with respect to the reference simulation as error $=\frac{\left|X_{w c}-X_{w f}\right|}{X_{w f}}$ where $X_{\mathrm{wc}}$ and $X_{\mathrm{wf}}$ are the mole fractions of species at the wall for coarse and fine grids, respectively. Figure 5 shows the order of accuracy of the new developed boundary condition with respect to the reference simulation results. Figure 5 demonstrates that the error reduces as the time step decreases and the calculated slope is 1.93 , which is very close to 2 . Therefore, the model is second order accurate with respect to time and space, and the new modifications do not deteriorate the order of accuracy. The present boundary condition shows second order accuracy for flat walls; however, it is expected to be first order accurate for the general case of curved walls.

\section{A. Simulation of steady catalytic combustion}

We consider initially a relatively low reaction rate. Equation (20) is used with $T_{w}=1200 \mathrm{~K}, E_{a}=77 \mathrm{~kJ} / \mathrm{mol}$, and $A_{0}=1.27 \times 10^{5} \mathrm{~cm} / \mathrm{s}$, as in [14]. To increase or decrease the

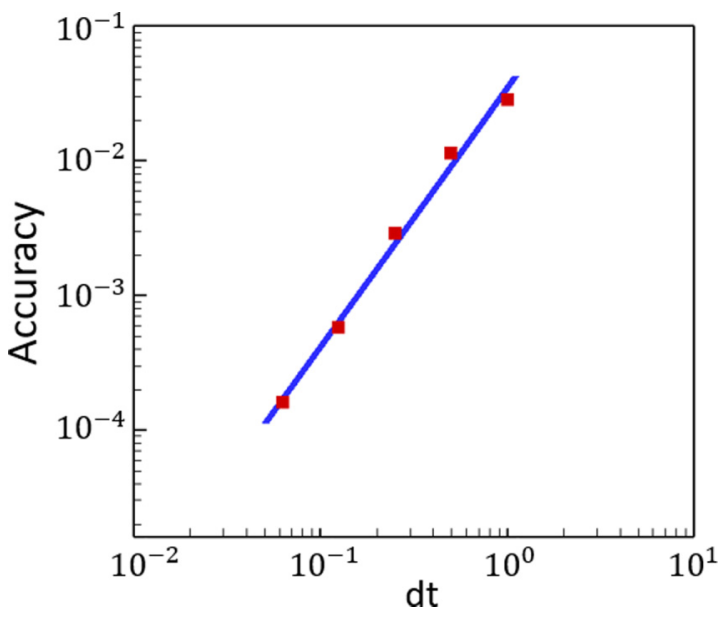

FIG. 5. The present model's order of convergence. reaction rate, we multiply $A_{0}$ by a desirable factor. Figure 6 illustrates the steady-state mole fraction profiles across the channel at $x=2 \mathrm{~mm}$ with $A=5.0 \times A_{0}$. With this choice of reaction rate, $\mathrm{Da}_{\mathrm{s}}=0.03$ and the system is mainly controlled by kinetics. As Fig. 6 shows, both the original and new models give accurate results (less than $3.5 \%$ difference) compared to the CFD results, although the surface Damköhler number in this simulation is five times higher than the one used in the previous study [14]. Under this condition, the diffusion coefficient in Eq. (24) is much smaller than the reaction rate coefficient. It is further noted that the implementation of the LB model is $O\left(u^{3}\right)$ accurate. Figure 7 also shows axial profiles of species mole fractions from $x=0$ until $x=2.5 \mathrm{~mm}$ along the symmetry plane $(z=0)$. It is evident that the mole fractions of species are also in good agreement not only at the particular cross section of Fig. 6 but also along the entire channel.

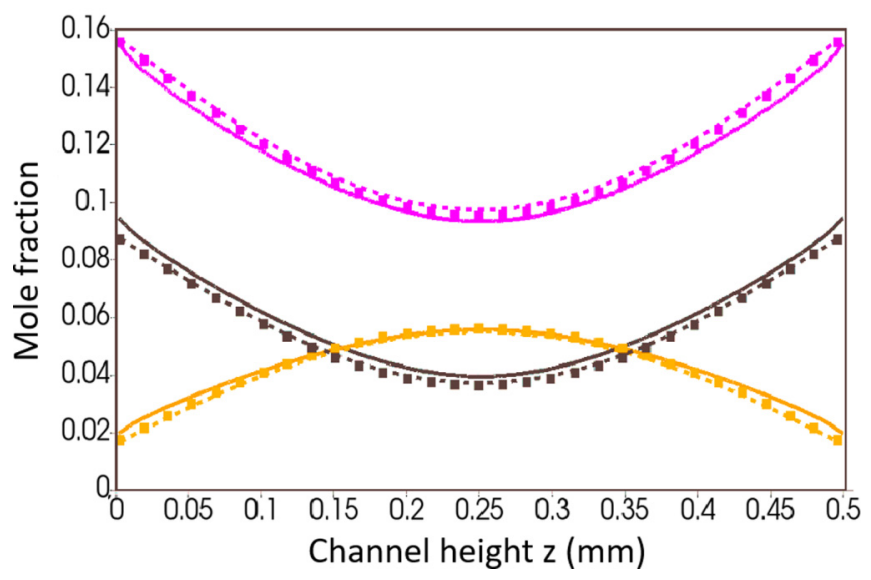

FIG. 6. Comparisons of mole fraction transverse profiles obtained from the old and present catalytic LB models as well as CFD simulations for a kinetically controlled reaction $\left(\mathrm{Da}_{\mathrm{s}}=0.03\right)$ at $x=2 \mathrm{~mm}$. 


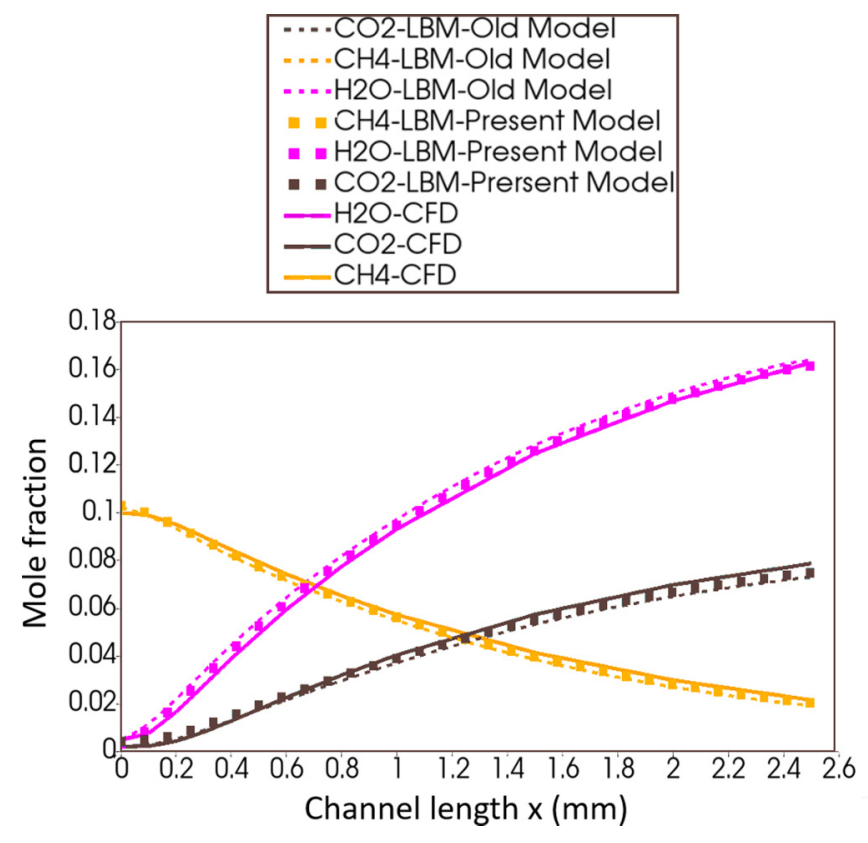

FIG. 7. Comparison of mole fraction axial profiles obtained from the old and present LB models as well as CFD results for kinetically controlled reaction $\left(\mathrm{Da}_{\mathrm{s}}=0.03\right)$ at the channel midplane.

In the second comparison case, the reaction rate coefficient is increased by a factor of $350\left(A=350 \times A_{0}, \mathrm{Da}_{\mathrm{s}}=2\right)$. The results are plotted in Figs. 8 and 9. It is evident that there is discrepancy between the original model results and the CFD simulations, which comes from the inaccurate calculation of the reaction rate. In fact, when the effect of diffusion is taken into account (in the new model) the denominator in Eq. (24) increases, hence, the reaction rate drops. When $\mathrm{Da}_{\mathrm{s}}>1$, one cannot neglect the effect of diffusion since the reaction rate calculated by Eq. (24) is different from the one obtained via Eq. (19). In contrast to the original model, the new reaction rate equation guarantees a positive numerator at different grid sizes and time steps. If the reaction rate coefficient increases further, for example five times more $\left(A=1750 \times A_{0}\right)$, the original catalytic reactive boundary condition model fails; however, the new model gives accurate results.

After comparison and validation of the present model, four simulations are carried out at different $\mathrm{Da}_{\mathrm{s}}$ numbers. Figure 10 illustrates the variation of molar fractions of $\mathrm{CH}_{4}$ and $\mathrm{CO}_{2}$ along the channel for $\mathrm{Da}_{\mathrm{s}}=0.0055,0.55,5.5,555$. The mole fraction axial profiles along the midplane of the channel and the mole fraction 2D maps are shown in this figure. However, it is difficult to distinguish between different reaction rates by only the methane mole fraction since its value is close to zero on the wall and the change would be too small. The contour plots demonstrate how fast the reaction occurs. According to the $\mathrm{CO}_{2}$ mole fraction contour, it is obvious that its production is much larger for higher reactivity cases. In spite of this, $\mathrm{CO}_{2}$ does not appreciably change as the reaction rate coefficient increases, especially when the system approaches the transport-limited regime $\left(\mathrm{Da}_{\mathrm{s}} \gg 1\right)$.

The original model could show relatively accurate results for cases of moderate reaction rates $\left(\mathrm{Da}_{\mathrm{s}}<1\right)$ provided that the grid size and time step are small enough. Nonetheless,

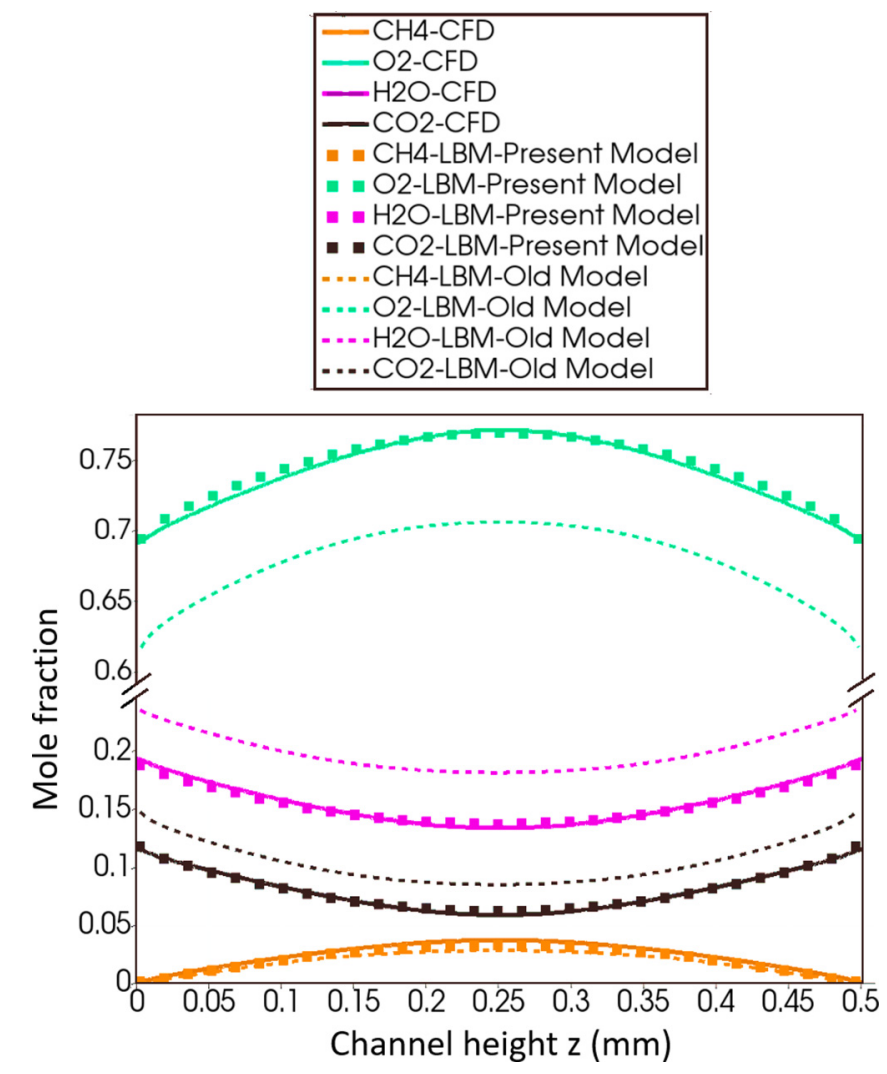

FIG. 8. Comparison of mole fraction transverse profiles obtained from the old and present LB models as well as CFD results for $\mathrm{Da}_{\mathrm{s}}=2$ at $x=2 \mathrm{~mm}$.

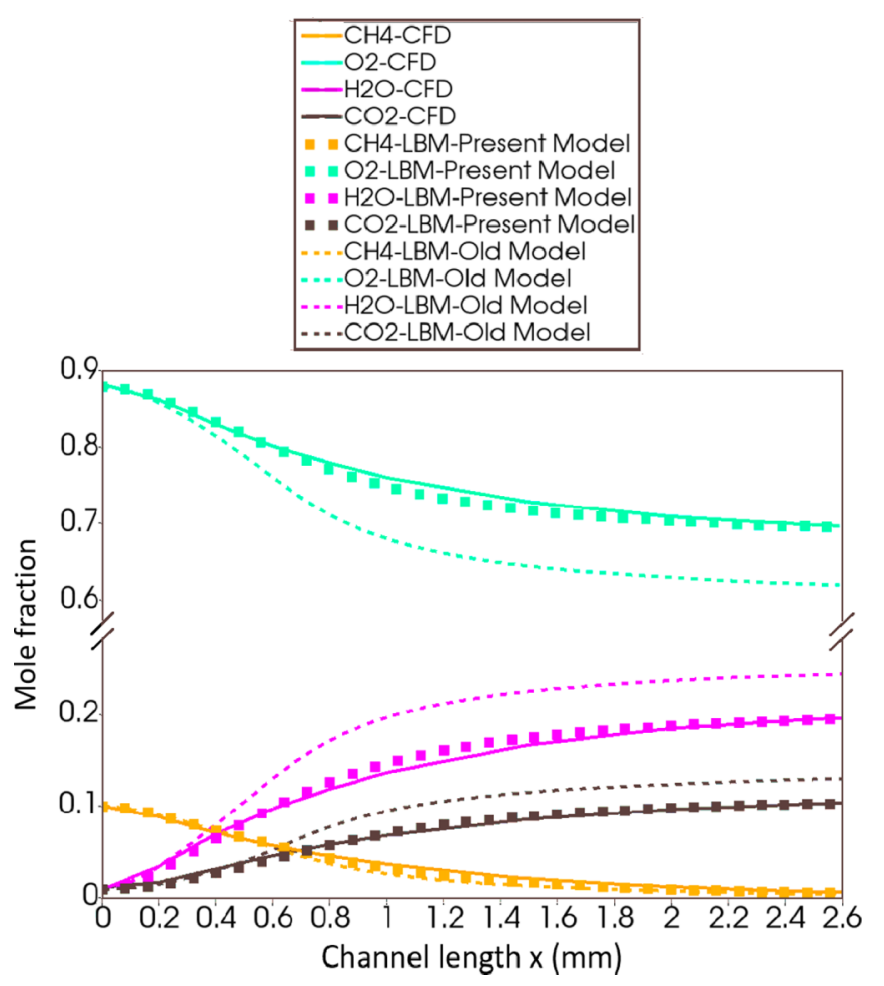

FIG. 9. Comparison of mole fraction axial profiles obtained from the old and present LB models as well as CFD results for $\mathrm{Da}_{\mathrm{s}}=2$ at the channel midplane. 
TABLE I. The effect of grid refinement on the accuracy of the original model $\mathrm{Da}_{\mathrm{s}}=4$. BC, boundary condition.

\begin{tabular}{llcr}
\hline \hline Numerical method & $l \times h \times w\left(l u^{3}\right)$ & $\mathrm{CH}_{4}$ mole fraction & $\frac{\left|X_{\mathrm{LBM}}-X_{\mathrm{CFD}}\right|}{X_{\mathrm{CFD}}}(\%)$ \\
\hline Old catalytic BC & $2000 \times 200 \times 5$ & 0.000089 & 38.6 \\
Old catalytic BC & $4000 \times 400 \times 5$ & 0.000125 & 13.79 \\
Old catalytic BC & $8000 \times 800 \times 5$ & 0.0001510 & 4.12 \\
Present catalytic BC & $2000 \times 200 \times 5$ & 0.0001706 & 17.65 \\
Present catalytic BC & $4000 \times 400 \times 5$ & 0.0001420 & 2.06 \\
CFD & $4000 \times 400 \times 1$ & 0.000145 & \\
\hline \hline
\end{tabular}
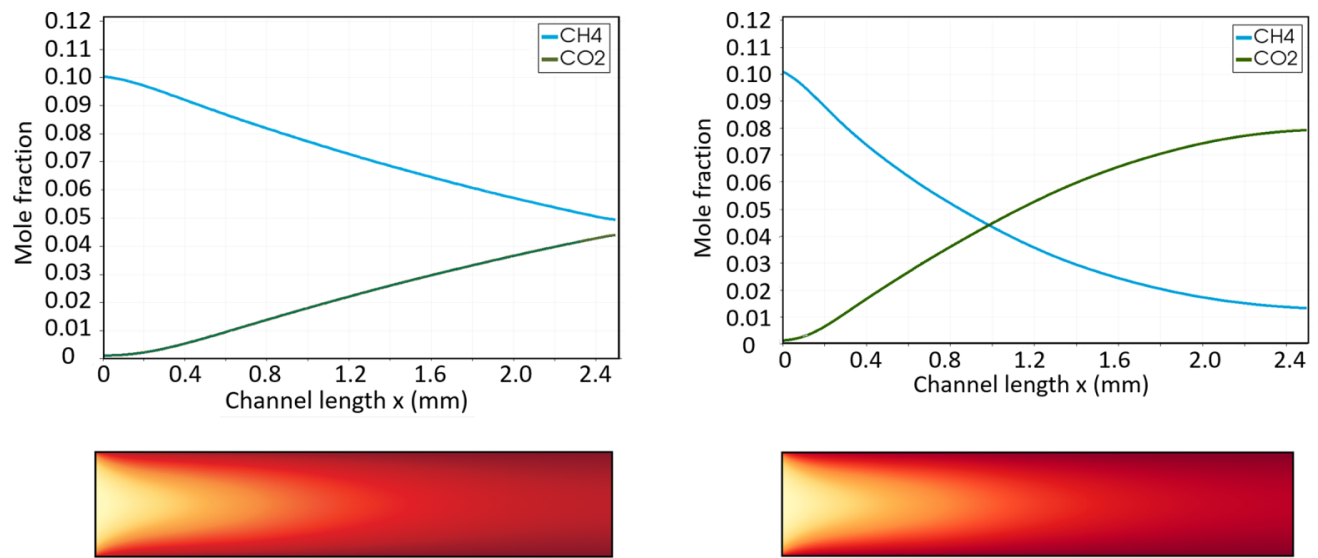

$\mathrm{CH}_{4}$ Mole Fraction

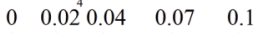

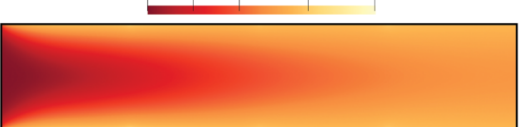

$\mathrm{CH}_{4}$ Mole Fraction

$\begin{array}{lllll}0 & 0.02 & 0.04 & 0.07 & 0.1\end{array}$

$\mathrm{CO}_{2}$ Mole Fraction

$\begin{array}{llllll}0 & 0.04 & 0.07 & 0.1 & 0.15\end{array}$

(a)
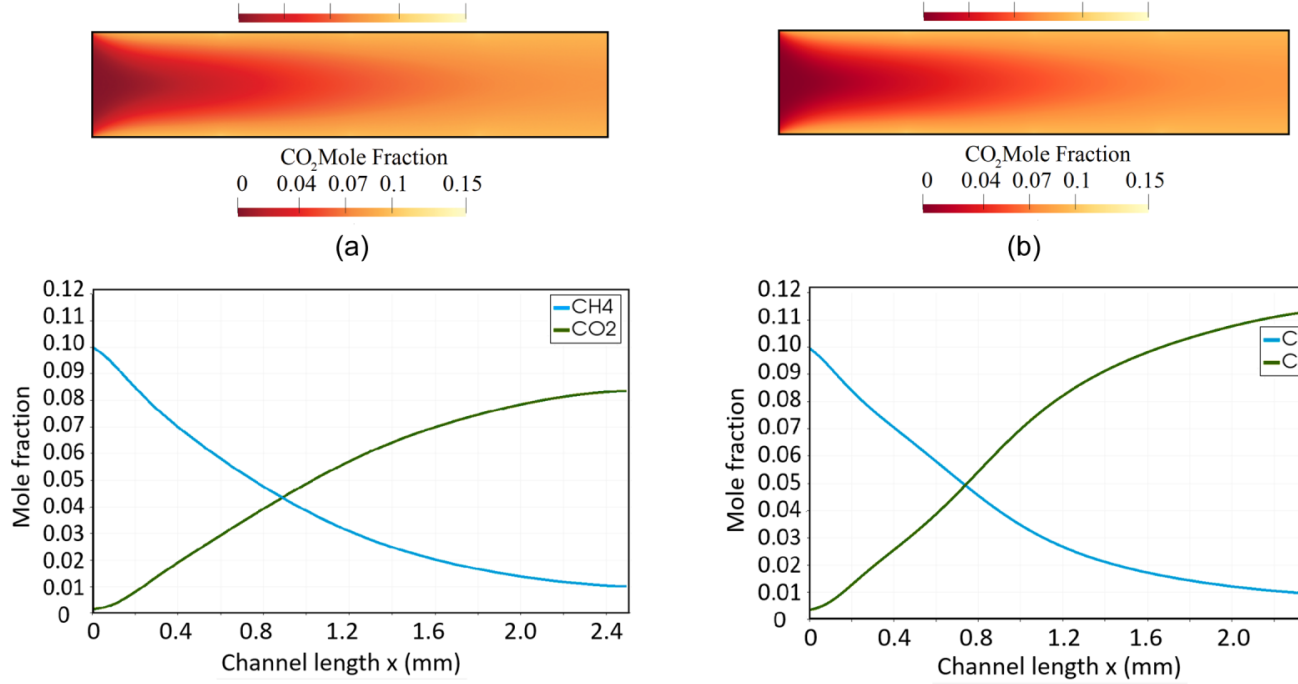

(b)
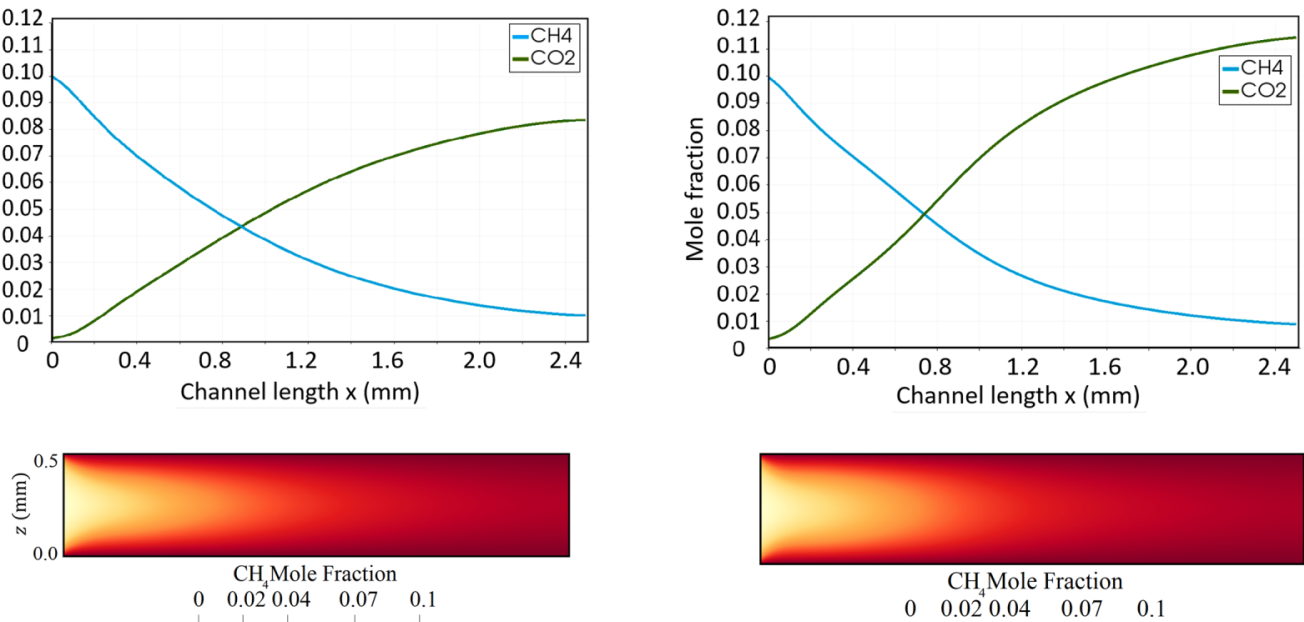

$\mathrm{CH}_{4}$ Mole Fraction

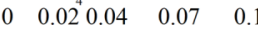

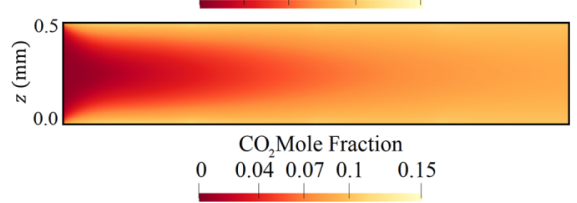

(c)

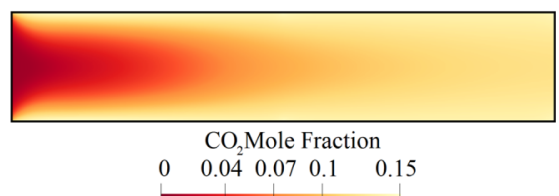

(d)

FIG. 10. The impact of Damköhler number on consumption and production of $\mathrm{CH}_{4}$ and $\mathrm{CO}_{2}: \mathrm{Da}_{\mathrm{s}}=0.0055,0.55,5.5,555$. 
it requires much smaller grid size when $\mathrm{Da}_{\mathrm{s}}>1$. Table I reports the effect of grid size on $\mathrm{CH}_{4}$ wall mole fraction at $x=1.5 \mathrm{~mm}$ obtained from the original and new LB catalytic models for $\mathrm{Da}_{\mathrm{s}}=4$. The difference compared to the CFD result reduces with grid refinement. Hence, by increasing the surface reactivity, the original model requires finer grid to have similar accuracy. It turns out that very costly simulations are needed or it is even practically impossible to simulate very high reactivity cases $\left(\mathrm{Da}_{\mathrm{s}}>10\right)$.

It is stressed that practical catalytic reactors are designed to operate at $\mathrm{Da}_{s} \gg 1$, so as to take full advantage of the available reactor length. For example, in the high-pressure channel-flow catalytic combustion of $\mathrm{H}_{2}$ and air over Pt [38] and of $\mathrm{CH}_{4}$ over Pt [40], $\mathrm{Da}_{\mathrm{s}}$ up to 180 and 102 are obtained, respectively. Moreover, for dissolution-precipitation reactions occurring in geochemically reactive flows [21,22], calcite is dissolved in the presence of $\mathrm{HCl}$ acid. In such systems, the wormhole phenomena as well as the face dissolution are observed at $\mathrm{Da}_{\mathrm{s}}>10^{5}$ and $>10^{6}$, respectively. Hence, the presently developed model is suitable for simulating realistic catalytic and surface reaction dominated systems.

Conversely, the new model is capable of simulating all Damköhler numbers and shows more accurate results with the same grid size. The new model is computationally more efficient than the original model by a factor of $\sim 2^{d} \times N$ for Damköhler numbers $O(1)$, where $d$ is the spatial dimension $(d=2$ or 3$)$ and $N$ is the number of species.

The present catalytic model can predict mole fraction transverse profiles at very large Damköhler numbers (e.g., $\mathrm{Da}_{\mathrm{s}}>10^{3}$ ), which is practically impossible with the original catalytic model due to excessively high computational costs. Figure 11 illustrates $\mathrm{CH}_{4}$ and $\mathrm{CO}_{2}$ transverse profiles for three Damköhler numbers $10^{3}, 10^{6}$, and $10^{9}$ at $x=0.5 \mathrm{~mm}$. Such very high Damköhler numbers can be relevant in practical industrial systems, for example, the total oxidation of hydrocarbons and syngas fuels in power generation devices [37]. A good agreement between the present LB model simulations and the CFD results is observed, demonstrating the capability of the new catalytic model at very large Damköhler numbers. All LB simulations in Fig. 11 were made with $4000 \times 400 \times 5$ nodes. It is stressed that the old model could not converge even for the lowest $\mathrm{Da}_{\mathrm{s}}=10^{3}$ of Fig. 11 when using the largest possible grid size in our cluster (maximum $1600 \times 16000 \times 5$ grid points).

\section{B. Transient simulations}

It is quite demanding to evaluate differences in transient simulations since every node along the reactive wall exhibits different reaction rates due to the combined effects of convection, diffusion, and reaction. Figure 12 shows the methane mole fraction difference at the wall at two locations, $x=0.00125 \mathrm{~mm}$ and $x=2.0 \mathrm{~mm}$, predicted by the two catalytic reactive models for $\mathrm{Da}_{\mathrm{s}}=2$.

In order to assess the difference in these two locations, we can rewrite Eq. (24) in terms of an effective reaction rate coefficient as $\dot{S}_{1}=k_{\mathrm{eff}} C_{1 n}$. While the difference in $k_{\mathrm{eff}}$ between the two catalytic models is almost independent of node location (by assuming a constant diffusion coefficient everywhere
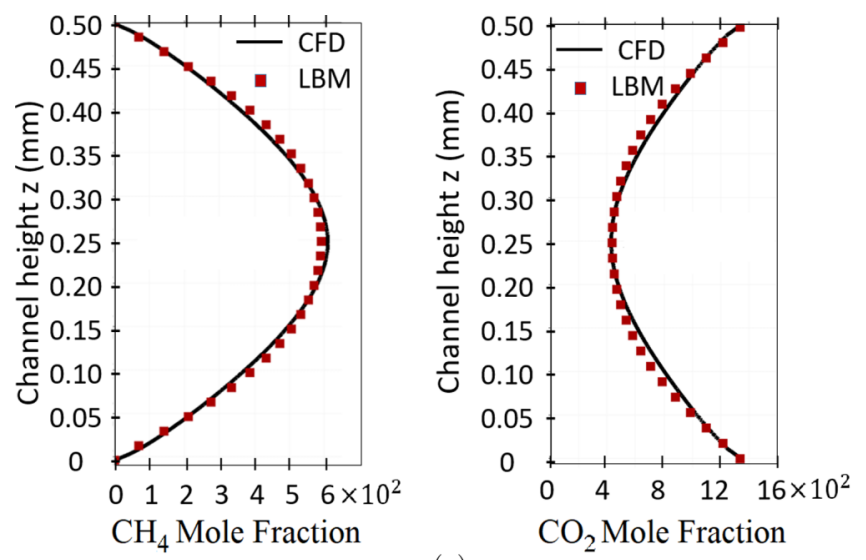

(a)
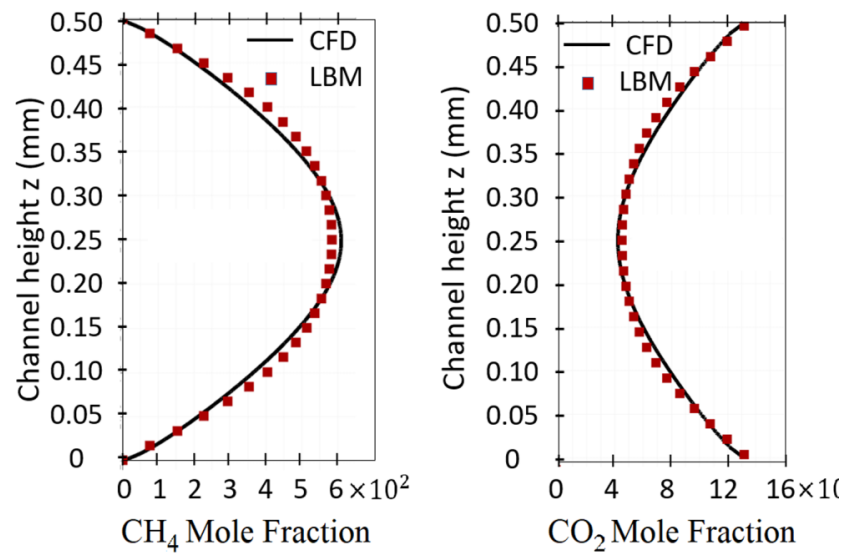

(b)
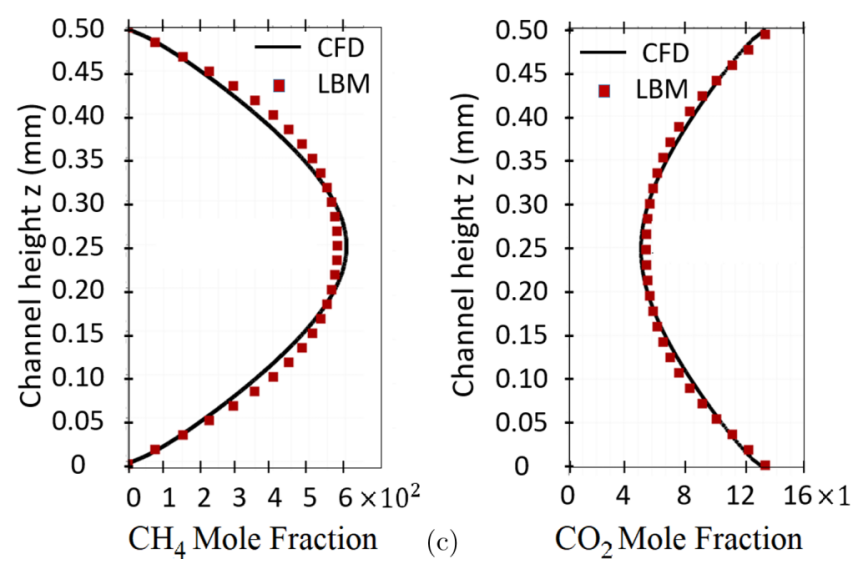

FIG. 11. Comparison of mole fraction transverse profiles obtained from the present catalytic LB model and CFD results at high Damköhler numbers $(x=0.5 \mathrm{~mm})(\mathrm{a}) \mathrm{Da}_{\mathrm{s}}=10^{3}$, (b) $\mathrm{Da}_{\mathrm{s}}=10^{6}$, and (c) $\mathrm{Da}_{\mathrm{s}}=10^{9}$.

in the channel) and remains constant during simulation, $C_{1 n}$ decreases in a different way. In terms of absolute values, the mole fractions of reactive nodes near the inlet show larger deviation from the original model (up to 90\%) because the initial mole fraction of methane (0.1) is fed by the inlet, and hence, the error comes only from the reaction rate coefficient calculated by two models. Therefore, all reactive nodes show up to $90 \%$ difference at the initial time. 


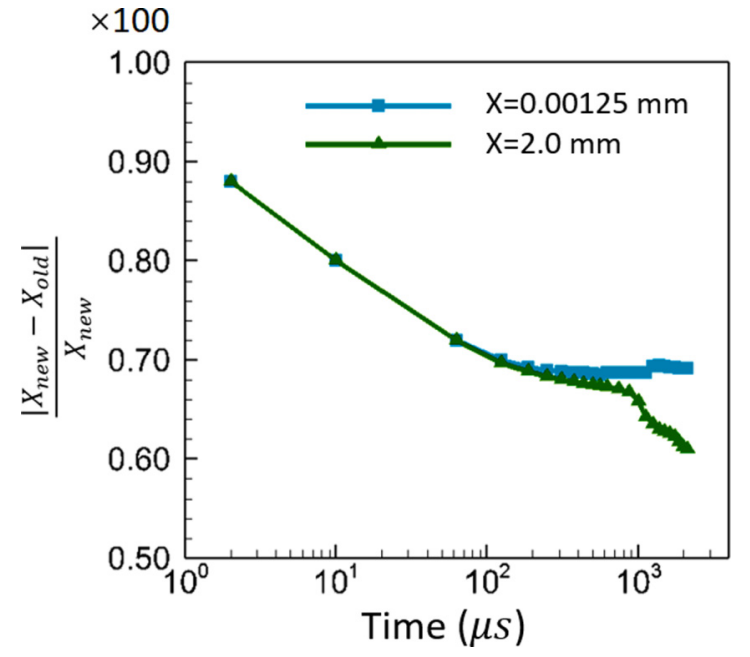

FIG. 12. The error in the $\mathrm{CH}_{4}$ mole fraction obtained by the old and the present catalytic models in transient simulation at two axial locations on the channel wall.

However, the difference is not fixed as time progresses since the mole fractions next to reactive nodes $\left(C_{1 n}\right)$ change. Therefore, the difference is a function of node position. For instance, $x=0.00125 \mathrm{~mm}$ is very close to the inlet, hence, the error reduces to $69 \%$ after a short time. But farther downstream (e.g., $x=2.0 \mathrm{~mm}$ ), the mole fraction decreases more after some time because a large portion of the inlet methane has been consumed upstream, and there is less methane left to react. Consequently, the reaction rate decreases along the $x$ direction.

Contrary to the $k_{\text {eff }}$ which is fixed and causes more mass consumption due to inaccurate reaction rate calculated by the old model, the concentration is lower than the actual value (since more methane is consumed upstream) leading to less mass consumption. Therefore, some part of the error calculated by Eq. (19) is canceled out. For this reason, we see less differences between mole fractions obtained by two catalytic models at steady state compared to the initial times. Nevertheless, the percentage of the error in the bulk flow increases at downstream locations due to accumulation of errors from the upstream reactive nodes (see also Fig. 9).

\section{CONCLUSIONS}

The kinetic model for isothermal multispecies flows with heterogeneous catalytic reactions at wall boundaries which was proposed in Ref. [13] has been extended to describe flows with arbitrary catalytic reactivity (Damköhler numbers, $\mathrm{Da}_{\mathrm{s}}$, spanning from zero to infinity). It is shown that the limitations of the old catalytic reactive boundary condition [13] stem from its local nature (constructed using on-wall node information) and the ensuing increasing inaccuracies with decreasing wall concentration of the deficient reactant as $\mathrm{Da}_{\mathrm{s}}$ increases. Simulations in a catalytic isothermal straight channel and comparisons with a CFD code indicate that the old model provides accurate solutions for $0<\mathrm{Da}_{\mathrm{s}}<1$, a range that encompasses the kinetically controlled regime and the low end of the mixed transport-to-kinetics controlled regime. The new macroscopic catalytic boundary condition model invokes nonlocal information by appropriately replacing the wall concentration of the deficient reactant with that of the near-wall gas node. The constructed model is second order accurate with respect to time and space. Simulations are carried out with the new and old LB catalytic models and with a CFD code for the total oxidation of methane in a straight channel-flow geometry. An irreversible catalytic reaction is considered, first order with respect to the deficient methane reactant. The comparisons indicate that the new model, even with a 16fold reduction in the number of nodes (for a $2 \mathrm{D}$ case with four species), attains better accuracy than the old model at $\mathrm{Da}_{\mathrm{s}}=4$. More importantly, at $\mathrm{Da}_{\mathrm{s}}>10^{3}$ (strongly transportcontrolled regime) where the old model could not converge for all practical node numbers, the new model provided accurate results. It was thus shown that the new model could successfully simulate many practical systems with high Damköhler numbers, such as the deep oxidation of hydrocarbons over noble metal catalysts in power generation devices. Finally, the new catalytic boundary condition can be implemented to other reactive LB models for the efficient simulation of high Damköhler number flows.

\section{ACKNOWLEDGMENTS}

We acknowledge support from the Swiss National Science Foundation (SNSF) under Project No. 200021_179019 and by the Swiss Supercomputing Center CSCS under projects GPUs92, s1010 and psi03.
[1] S. Succi, G. Smith, and E. Kaxiras, Lattice Boltzmann simulation of reactive microflows over catalytic surfaces, J. Stat. Phys. 107, 343 (2002).

[2] Q. Kang, D. Zhang, and S. Chen, Simulation of dissolution and precipitation in porous media, J. Geophys. Res. Solid Earth 108, 2505 (2003).

[3] X. Shan and G. Doolen, Diffusion in a multicomponent lattice Boltzmann equation model, Phys. Rev. E 54, 3614 (1996).

[4] L. S. Luo and S. S. Girimaji, Theory of the lattice Boltzmann method: Two-fluid model for binary mixtures, Phys. Rev. E 67, 036302 (2003).
[5] M. E. McCracken and J. Abraham, Lattice Boltzmann methods for binary mixtures with different molecular weights, Phys. Rev. E 71, 046704 (2005).

[6] P. Asinari, Semi-implicit-linearized multiple-relaxation-time formulation of lattice Boltzmann schemes for mixture modeling, Phys. Rev. E 73, 056705 (2006).

[7] S. Arcidiacono, I. V. Karlin, J. Mantzaras, and C. E. Frouzakis, Lattice Boltzmann model for the simulation of multicomponent mixtures, Phys. Rev. E 76, 046703 (2007).

[8] S. Succi, G. Bella, and F. Papetti, Lattice kinetic theory for numerical combustion, J. Sci. Comput. 12, 395 (1997). 
[9] A. Gabrielli, S. Succi, and E. Kaxiras, A lattice Boltzmann study of reactive microflows, Comput. Phys. Commun. 147, 516 (2002).

[10] X. He, N. Li, and B. Goldstein, Lattice Boltzmann simulation of diffusion-convection systems with surface chemical reaction, Mol. Simul. 25, 145 (2000).

[11] Q. Kang, P. C. Lichtner, and D. Zhang, An improved lattice Boltzmann model for multicomponent reactive transport in porous media at the pore scale, Water Resour. Res. 43, W12S14 (2007).

[12] L. Li, R. Mei, and J. F. Klausner, Lattice Boltzmann models for the convection-diffusion equation: D2Q5 vs D2Q9, Int. J. Heat Mass Transfer 108, 41 (2017).

[13] S. Arcidiacono, J. Mantzaras, and I. V. Karlin, Lattice Boltzmann simulation of catalytic reactions, Phys. Rev. E 78, 046711 (2008).

[14] J. Kang, N. I. Prasianakis, and J. Mantzaras, Thermal multicomponent lattice Boltzmann model for catalytic reactive flows, Phys. Rev. E 89, 063310 (2014).

[15] Q. Kang, P. C. Lichtner, H. S. Viswanathan, and A. I. AbdelFattah, Pore scale modeling of reactive transport involved in geologic $\mathrm{CO}_{2}$ sequestration, Transp. Porous Media 82, 197 (2010).

[16] E. Chiavazzo, I. V. Karlin, A. N. Gorban, and K. Boulouchos, Coupling of the model reduction technique with the lattice Boltzmann method for combustion simulations, Combust. Flame 157, 1833 (2010).

[17] A. F. Di Rienzo, P. Asinari, E. Chiavazzo, N. I. Prasianakis, and J. Mantzaras, Lattice Boltzmann model for reactive flow simulations, Europhys. Lett. 98, 34001 (2012).

[18] Q. Kang and P. C. Lichtner, A lattice Boltzmann method for coupled fluid flow, solute transport, and chemical reaction, in Progress in Computational Physics Vol. 3: Novel Trends in Lattice-Boltzmann Methods, edited by M. Ehrhardt (Bentham Science Publishers, Sharjah, 2013).

[19] J. Kang, N. I. Prasianakis, and J. Mantzaras, Lattice Boltzmann model for thermal binary-mixture gas flows, Phys. Rev. E 87, 053304 (2013).

[20] C. Lin and K. H. Luo, MRT discrete Boltzmann method for compressible exothermic reactive flows, Comput. Fluids 166, 176 (2018).

[21] S. Molins, C. Soulaine, N. I. Prasianakis, A. Abbasi, P. Poncet, A. J. Ladd, V. Starchenko, S. Roman, D. Trebotich, H. A. Tchelepi et al., Simulation of mineral dissolution at the pore scale with evolving fluid-solid interfaces: Review of approaches and benchmark problem set, Comput. Geosci. 1, 34 (2020).

[22] N. I. Prasianakis, M. Gatschet, A. Abbasi, and S. V. Churakov, Upscaling strategies of porosity-permeability correlations in reacting environments from pore-scale simulations, Geofluids 2018, 14688123 (2018).

[23] R. Sui, J. Mantzaras, E.-T. Es-Sebbar, M. A. Safi, and R. Bombach, Impact of gaseous chemistry in $\mathrm{H}_{2} / \mathrm{O}_{2} / \mathrm{N}_{2}$ combustion over platinum at fuel-lean stoichiometries and pressures of 1.0-3.5 bar, Energy Fuels 31, 11448 (2017).

[24] L. Jv, C. Zhang, and Z. Guo, Local reactive boundary scheme for lattice Boltzmann method, arXiv:1904.02133 [Comput. Physi. (2019).
[25] N. Kulyk, D. Berger, A. S. Smith, and J. Harting, Catalytic flow with a coupled finite difference-Lattice Boltzmann scheme, Comput. Phys. Commun. 256, 107443 (2020).

[26] N. I. Prasianakis, T. Rosén, J. Kang, J. Eller, J. Mantzaras, and F. N. Büchi, Simulation of 3D porous media flows with application to polymer electrolyte fuel cells, Commun. Comput. Phys. 13, 851 (2013).

[27] S. Arcidiacono, S. Ansumali, I. V. Karlin, J. Mantzaras, and K. B. Boulouchos, Entropic lattice Boltzmann method for simulation of binary mixtures, Math. Comput. Simul. 72, 79 (2006).

[28] R. B. Bird, W. E. Stewart, E. N. Lightfoot, and D. J. Klingenberg, Introductory Transport Phenomena (Wiley, New York, 2015).

[29] C. R. Wilke, A viscosity equation for gas mixtures, J. Chem. Phys. 18, 517 (1950).

[30] S. Ansumali and I. V. Karlin, Kinetic boundary conditions in the lattice Boltzmann method, Phys. Rev. E 66, 026311 (2002).

[31] R. W. Schefer, Catalyzed combustion of $\mathrm{H}_{2}$ /air mixtures in a flat plate boundary layer: II. Numerical model, Combust. Flame 45, 171 (1982).

[32] M. Reinke, J. Mantzaras, R. Schaeren, R. Bombach, A. Inauen, and S. Schenker, High-pressure catalytic combustion of methane over platinum: In situ experiments and detailed numerical predictions, Combust. Flame 136, 217 (2004).

[33] J. Mantzaras, R. Sui, C. K. Law, and R. Bombach, Heterogeneous and homogeneous combustion of fuel-lean $\mathrm{C}_{3} \mathrm{H}_{8} / \mathrm{O}_{2} / \mathrm{N}_{2}$ mixtures over rhodium at pressures up to 6 bar, Proc. Combust. Inst. 38, 6473 (2021).

[34] J. Mantzaras and C. Appel, Effects of finite rate heterogeneous kinetics on homogeneous ignition in catalytically stabilized channel flow combustion, Combust. Flame 130, 336 (2002).

[35] T. Krüger, H. Kusumaatmaja, A. Kuzmin, O. Shardt, G. Silva, and E. M. Viggen, The Lattice Boltzmann Method (Springer, New York, 2017).

[36] R. J. Kee, D.-L. Graham, J. Warnatz, M. E. Coltrin, J. A. Miller, and H. K. Moffat, A fortran computer code package for the evaluation of gas-phase, multicomponent transport properties, Report No. SAND86-8246B, Sandia National Laboratories (1996).

[37] J. Mantzaras, Progress in non-intrusive laser-based measurements of gas-phase thermoscalars and supporting modeling near catalytic interfaces, Prog. Energy Combust. Sci. 70, 169 (2019).

[38] Y. Ghermay, J. Mantzaras, R. Bombach, and K. Boulouchos, Homogeneous combustion of fuel-lean $\mathrm{H}_{2} / \mathrm{O}_{2} / \mathrm{N}_{2}$ mixtures over platinum at elevated pressures and preheats, Combust. Flame 158, 1491 (2011).

[39] M. Coltrin, R. Kee, and F. Rupley, Surface Chemkin: A fortran package for analyzing heterogeneous chemical kinetics at the solid surface-gas phase interface, Report No. SAND90-8003C, Sandia National Laboratories (1996).

[40] M. Reinke, J. Mantzaras, R. Bombach, S. Schenker, and A. Inauen, Gas phase chemistry in catalytic combustion of methane/air mixtures over platinum at pressures of 1 to 16 bar, Combust. Flame 141, 448 (2005). 